\title{
BMJ Open Randomised controlled trial to assess the impact of a lifestyle intervention (ActWELL) in women invited to NHS breast screening
}

\author{
Annie S Anderson, ${ }^{1}$ Angela Mary Craigie, ${ }^{1}$ Stephanie Gallant, ${ }^{1}$ Chloe McAdam, ${ }^{2}$ \\ E Jane Macaskill, ${ }^{3}$ Nanette Mutrie, ${ }^{2}$ Aileen Rae Neilson, ${ }^{4}$ Ronan E O'Carroll, ${ }^{5}$ \\ Petra Rauchhaus, ${ }^{6}$ Naveed Sattar, ${ }^{7}$ Martine Stead, ${ }^{8}$ Shaun Treweek ${ }^{9}$
}

To cite: Anderson AS, Craigie AM, Gallant S, et al. Randomised controlled trial to assess the impact of a lifestyle intervention (ActWELL) in women invited to NHS breast screening. BMJ Open 2018;8:e024136. doi:10.1136/ bmjopen-2018-024136

- Prepublication history and additional material for this paper are available online. To view these files, please visit the journal online (http://dx.doi. org/10.1136/bmjopen-2018024136).

Received 10 May 2018 Revised 13 September 2018 Accepted 21 September 2018

Check for updates

(C) Author(s) (or their employer(s)) 2018. Re-use permitted under CC BY-NC. No commercial re-use. See rights and permissions. Published by BMJ.

For numbered affiliations see end of article.

Correspondence to

Professor Annie S Anderson; a.s.anderson@dundee.ac.uk

\section{ABSTRACT}

Introduction In Scotland, the incidence of breast cancer is predicted to rise significantly in the next few decades and while there are measures to support reductions in morbidity and mortality, the breast cancer community is currently exploring preventative opportunities including supporting weight management programmes in postmenopausal women. This study aims to assess the effectiveness and cost-effectiveness of a theorybased, community delivered, minimal contact, weight management (diet, physical activity and behaviour change techniques) programme (ActWELL) in women with a body mass index $(\mathrm{BMI})>25 \mathrm{~kg} / \mathrm{m}^{2}$ attending routine breast cancer screening appointments.

Methods and analysis The study will be a four-centre, 1:1 parallel group randomised controlled trial of a 12-month weight management intervention initiated in breast cancer screening centres, delivered by trained Breast Cancer Now lifestyle coaches in community settings. The intervention programme involves two intervention meetings with coaches plus (up to) nine telephone contacts over 12 months. The programme will focus on personalised diet (including alcoholic and sugary drinks) and physical activity habits. Behaviour change techniques include self-monitoring, goal setting, implementation intentions, action and coping plans. The study has a sample size of 414 women with a BMI $>25 \mathrm{~kg} / \mathrm{m}^{2}$ attending routine National Health Service breast cancer screening appointments. Measures will be taken at baseline, 12 weeks and at 12-month followup, complemented by qualitative interviews exploring perceived acceptability and impact on habitual behaviours The two co-primary outcomes are mean change in measured body weight and change in physical activity between groups to 12 months. Secondary outcomes are changes in eating habits, alcohol intake, sedentary time, quality of life, waist circumference, lipid, haemoglobin A1c and insulin profiles, blood pressure and cost-effectiveness of the intervention.

Ethics and dissemination The protocol has been approved by East of Scotland Research Ethics Committee (17/ES/0073). All participants provide written informed consent. Dissemination will be through peer-reviewed publication and conference presentations.

Trial registration number ISRCTN11057518; Pre-results.
Strengths and limitations of this study

- This work has the potential to provide routine support for weight management for women aged over 50 years.

- Multicentre, randomised controlled design.

- Novel approach to deliver weight management by trained volunteers in community locations.

- The study has a large potential reach with around $73 \%$ of ALL women of this age group attending appointments including high numbers from disadvantaged backgrounds.

- The intervention will not be readily accessible for women who do not accept screening appointments.

\section{INTRODUCTION}

Breast cancer accounted for $14.5 \%$ of all cancer diagnoses in Scotland in 2014 and accounts for $28 \%$ of female cancer cases. ${ }^{1}$ Incidence is increasing and current predictions from ISD (Scottish Government) suggest a rise by $27.5 \%$ between $2008-2012$ and 2023-2027. ${ }^{2}$

While many factors are implicated in aetiology (genetics, reproductive history, hormone use), current estimates suggest that $38 \%$ of breast cancers in postmenopausal women in the UK are related to physical inactivity, alcohol consumption and body fatness. ${ }^{3}$ There is consistent evidence that being overweight or obese throughout adulthood increases the risk of postmenopausal breast cancer. ${ }^{4}$

In addition, gaining weight in adult life is a strong predictor of breast cancer (especially in women who have not taken hormone replacement therapy). ${ }^{5}$ Ahn et al reported that at any body mass index (BMI), increased weight in adult life is associated with greater risk of breast cancer and a gain of $2-10 \mathrm{~kg}$ after the age of $50(\mathrm{eg}$, postmenopausal) is associated with a $30 \%$ 
increase in breast cancer risk. ${ }^{6}$ Findings from the European Prospective Investigation into Cancer and Nutrition (EPIC) study have also demonstrated that high weight gain in middle adulthood increases the risk of breast cancer. $^{7}$ In the Women's Health Initiative, Neuhouser et $a l^{8}$ reported that postmenopausal women with a BMI $<25 \mathrm{~kg} / \mathrm{m}^{2}$ at baseline who gained $>5 \mathrm{~kg}$ of body weight during the follow-up period (median 13 years) had a $36 \%$ increase risk of developing breast cancer.

Lifestyles and weight management are also related to risk reduction. Women meeting at least five of the World Cancer Research Fund prevention guidelines for lifestyle show a $60 \%$ lower risk for breast cancer compared with women meeting none of the guidelines ${ }^{9}$ and a recent systematic review reported that high versus low adherence to cancer prevention guidelines was associated with consistent reductions in breast cancer incidence. ${ }^{10}$ Data from audits of bariatric surgery show that large weight losses are associated with large decreases in female cancers. ${ }^{11}$ One recent North American study of 22198 people 3.5 years after bariatric surgery reported reductions in postmenopausal breast cancer of $42 \%{ }^{12}$ In addition, moderating weight gain in adult life through caloric adjustment and being physically active is likely to be of benefit for reduction in other cancers related to these behaviours ${ }^{13}$ as well as other non-communicable diseases. ${ }^{1415}$

Most (73\%) Scottish women aged 50-70 years accept invitations to attend the routine NHS Scottish Breast Screening Programme (NHSSBSP) and over 175000 women attend each year. ${ }^{16}$ In addition, women aged over 70 years are able to attend through self-referral. The NHSSBSP therefore provides a unique opportunity to endorse a weight management intervention. However, a greater understanding of the benefits, costs, acceptability and impacts are needed to examine whether a NHSSBSP-initiated intervention can be effective and cost-efficient.

In 2007, Fisher et $a l^{17}$ reported that most women attending breast screening clinics are interested in receiving lifestyle advice, and an updated paper reporting the view of 1803 women shows overwhelming support for receiving interventions through this setting. ${ }^{18}$ However, a review published in $2013^{19}$ reported that while the importance of weight management in breast and colorectal cancer prevention is widely recognised, there is little evidence that lifestyle is discussed within cancer screening settings. It was also noted that the lack of advocacy about health behaviour change may endorse poor health behaviours by creating a 'health certificate effect'. This issue may be particularly relevant for body weight, where a lack of guidance to visibly obese patients may imply a lack of medical concern. The cancer research 'gap analysis' reviews by Breast Cancer Campaign ${ }^{20}{ }^{21}$ highlighted the role of breast screening programmes as an opportunity for promoting cancer prevention activities, but noted the challenge of finding ways to support and facilitate women to achieve healthy ways of life.

In Scotland, the breast cancer community (government, charities and health professionals) is currently exploring innovative and sustainable preventative opportunities including supporting weight management programmes. The Scottish Health Survey ${ }^{22}$ has reported that $72 \%$ of women aged $55-74$ years have a BMI $>25 \mathrm{~kg} /$ $\mathrm{m}^{2}$ (76\% in women living in areas of higher deprivation). Furthermore, $42 \%$ of women do not achieve the recommendation of $150 \mathrm{~min}$ of physical activity per week, and this proportion increases with age. The National Institute of Clinical Excellence ${ }^{23}$ recommend that lifestyle weight management programmes are multicomponent and aim to reduce a person's energy intake and help them to be more physically active by changing behaviour. However, access to such National Health Service (NHS) programmes is limited and commercial programmes have cost implications for low-income adults.

It is recognised that partnerships between the NHS and the voluntary sector offer significant value for money and the potential for greater 'reach' of interventions into community settings. The recent Lancet series on obesity highlighted that, despite government efforts to reduce the prevalence of obesity, these approaches are insufficient to help adults who are currently overweight or obese. ${ }^{24}$ Innovative strategies beyond those currently delivered by health professionals are needed to increase capacity of delivery of weight management programmes.

Community lifestyle interventions initiated in the breast cancer screening setting are a largely unexplored area, although repeated triennial appointments offer unique opportunities for initiation and re-enforcement. ${ }^{25}$ This setting also provides an opportunity to engage with women from areas of higher deprivation $(63 \%, 71 \%$ and $76 \%$ of women from Scottish Index of Multiple Deprivation (SIMD) $1,2,3$ quintiles, respectively attend for screening). ${ }^{26}$

The ActWELL feasibility study demonstrated that recruitment, retention, indicative results and participant acceptability merited a full randomised controlled trial to test the long-term impact of the intervention. In addition, $31 \%$ of participants recruited were from the lowest two quintiles of deprivation ${ }^{27}$ indicating significant potential to reach women from higher rates of social deprivation who also tend to be more obese.

Feedback from screening centre users showed while many were aware of lifestyle issues in relation to diabetes and cardiovascular disease, information about lifestyle and breast cancer was new and considered 'motivating' if the focus was on positive ways to help support behaviour change. ${ }^{28}$

The current study is designed to assess the effectiveness of a community-based, personalised, minimal contact weight management programme in women with a BMI $>25 \mathrm{~kg} / \mathrm{m}^{2}$ attending routine breast cancer screening clinics. The intervention programme is a collaboration between the charity Breast Cancer Now (BCN), NHSSBSP, local authority leisure centres and academic partners. This work is the first time that a cancer charity has offered volunteer capacity for cancer prevention action on weight management and offers significant potential to address gaps in public health efforts. The design is pragmatic to increase the relevance of the findings to policymakers, 
Women invited to take part in routine breast cancer screening will be given a brief PIS, and a verbal endorsement from NHSBSP staff. Those who are interested will leave contact details for the research team.

Alternatively women can call or email the study team directly.

Interested women will be telephone screened (for self-reported height and weight) and (if eligible) sent a full PIS and an appointment date to the research centre to provide informed consent and complete baseline

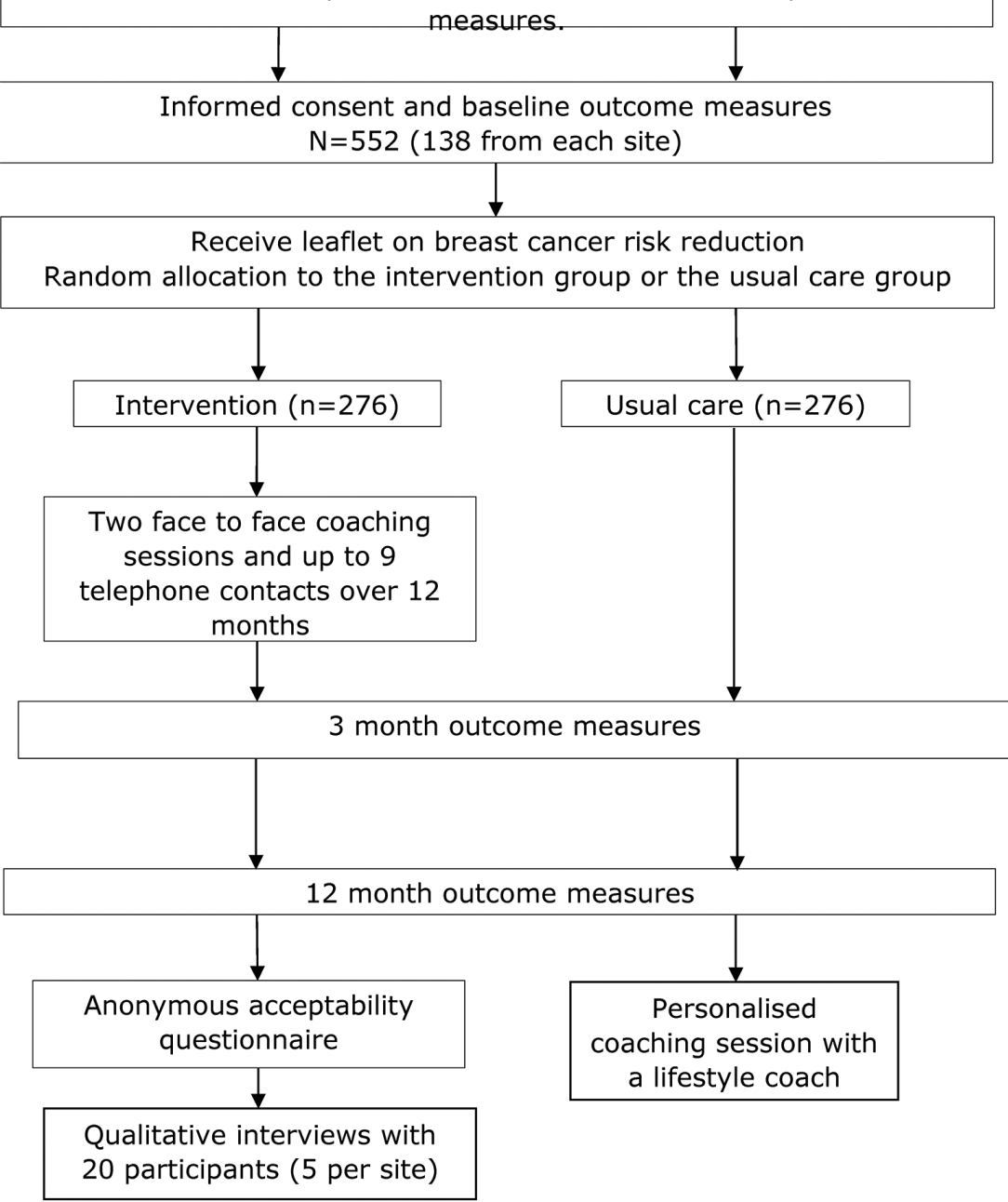

Figure 1 Study flow chart. NHSSBSP, NHS Scottish Breast Screening Programme; PIS, Participant Information Sheet.

women eligible for breast screening and health professionals (see online supplementary appendix 1 ).

The study aims to assess the effectiveness and cost-effectiveness of a theory-based, community delivered, minimal contact, weight management (diet, physical activity and behaviour change techniques) programme (ActWELL) in women with a $\mathrm{BMI}>25 \mathrm{~kg} / \mathrm{m}^{2}$ attending routine breast cancer screening clinics.

\section{METHODS AND ANALYSIS}

Trial design and setting

The studywill be afour-centre, 1:1 parallel-arm, randomised controlled trial of a 12-month, weight management intervention. The participants will be randomly allocated to one of two groups: (1) standard care with health information leaflet or (2) experimental group who receive the ActWELL intervention. Potential participants will be introduced to the study while attending a routine NHS breast cancer screening appointment (static and mobile screening units) in Scotland. The study participant flow is presented in figure 1.

All trial measurements will be undertaken by trained research nurses at baseline, 12 weeks and 12 months within the NHS Clinical Research Centres in the areas served by the breast screening sites.

The intervention is delivered and supported by BCN volunteers who have undergone 2 days bespoke training on the intervention. The face-to-face intervention 
communications are delivered within local authority run leisure centres (or other appropriate community locations).

\section{Participants}

All attendees at routine NHS breast screening appointments will be advised about the study by NHSSBSP staff in writing and verbally. On checking in at the clinic women will be given a brief study information leaflet by the receptionist. During their visit the mammographer will briefly (approximately $30 \mathrm{~s}$ ) mention the study verbally. If they are agreeable to a researcher making contact with them, women will be invited to leave their details on a contact card (telephone/email/postcode), which they can place in a study box in the reception area. A pop up banner, or poster, will also be displayed in the breast screening clinic or mobile van to further highlight the study and how to contact the research team. All NHS screening staff will be provided with training which introduces the aims of the study, why the trial is designed the way it is, the importance of their role, how to minimise the time taken to introduce the study and how to answer common questions. Within each site, a team 'champion' will be identified from within the NHSSBSP staff to encourage colleagues and coordinate recruitment efforts.

Research staff will contact women leaving contact cards within 2 weeks and assess study eligibility.

\section{Inclusion criteria}

- Attending, or invited to attend, routine breast screening clinics (not recall clinics).

- Measured BMI $>25 \mathrm{~kg} / \mathrm{m}^{2}$.

- Age 50-70 years.

\section{Exclusion criteria}

- Currently undergoing treatment for any malignant condition (excluding basal or squamous cell skin cancers).

- Reported contraindication to physical activity (eg, recent surgery).

- Reported contraindication to weight loss (eg, currently following a recovery programme for weight gain).

- On a specialised diet, for example, gluten free.

- Diagnosis of type 1 diabetes.

- Current use of insulin.

- No telephone contact.

- Unable to consent.

Participants who are considered eligible will be invited to attend their local research centre to provide informed consent before commencing baseline measures.

Participants found to be ineligible to take part, either on telephone screening or at a baseline visit, will be thanked for their time and will be offered, by post or email, lifestyle and cancer prevention information and/ or information on local leisure facilities applicable to them. Where possible, this will also apply to women who have expressed an interest (eg, left a completed card) but who have not been selected to take part in the trial due to the volume of people that can be seen by the research team. In the event of very large numbers of cards being returned, it may not be possible to contact all women and this is made clear in the brief information leaflet.

\section{Randomisation}

Participants will be randomised centrally at a 1:1 ratio into the intervention or usual care groups using the web-based TRuST system designed by Tayside Clinical Trials Unit (TCTU). Randomisation will be stratified by site and minimised by socioeconomic status based on SIMD $^{29}$ (two groups: SIMD 1 or 2; SIMD $\geq 3$ ).

In addition, a subgroup comprising 146 women (73 from each group) will be randomly allocated by the TRUST system to receive an activPAL monitor (accelerometer; PAL Technologies, Glasgow, UK) as an objective measure of physical activity. This number has been identified to meet statistical power (see below).

\section{Usual care group}

Following the end of their baseline measures, participants will receive a cancer prevention leaflet. ${ }^{30}$ The study administrator will then notify participants of their randomisation by letter. On completion of their 12-month follow-up visit, women will be offered a single personalised coaching session and the ActWELL intervention booklet.

\section{Intervention group}

Following the end of their baseline measures, participants will receive a cancer prevention leaflet. ${ }^{30}$ The study administrator will then notify participants of their randomisation by letter. The BCN volunteer coordinator will allocate a BCN lifestyle coach to the participant. The lifestyle coach will contact the participant to arrange an appointment for their first face-to-face visit.

\section{Blinding}

The study team will be blind to the participants' group allocation until completion of the primary outcome analysis. Exceptions are the trial manager, study administrator, lifestyle coaches and participants who cannot be blinded owing to the nature of the intervention.

\section{Intervention}

Initial work focused on optimising the intervention to scale up the previous prototype for a 12-month follow-up period. This was developed from the original feasibility study protocol and using feedback obtained from study participants and lifestyle coaches. Later feedback came from our recent research study offering a weight management lifestyle intervention to women attending family history clinics ${ }^{31}$ and our newly formed ActWELL public advisory team. The proposed study builds on existing behaviour change models, ${ }^{32}$ particularly the COM-B model. ${ }^{33}$ Thus, the intervention aims to incorporate capability for effective lifestyle change combined with opportunities for greater physical activity through an emphasis on walking initiatives and other community facilities (using 
taxonomy-derived effective behaviour change techniques $^{34}$ ) and increased motivation for weight management (through awareness raising within the teachable moment setting).

In addition, four formative qualitative focus groups with individuals in the target population will be held to obtain feedback on the prospective 12-month intervention including refinements on alcohol messaging.

\section{Intervention content}

The initial part of the intervention (1-3 months) will focus on helping achieve $7 \%$ of body weight loss (consistent with the lifestyle intervention in the highly effective Diabetes Prevention Programme ${ }^{35}$ ) and the remaining months will also combine techniques for weight loss maintenance (WLM) by addressing both caloric intake and energy expenditure.

Behavioural change techniques (BCT) include goal setting, implementation intentions, self-monitoring of body weight and feedback. The latter may be particularly important for WLM. This part of the intervention will take a habit formation-based approach using the 'Ten Top Tips' shown to be successful for WLM over a 2-year period. ${ }^{36}$ Social support will be encouraged throughout the 12 months through regular coach contact and encouragement to share the intervention with a friend/ buddy. The initial introduction to the leisure centre will also provide an introduction to locally available exercise facilities and classes.

Tailored personalised advice is a key component of all aspects of the intervention. Motivational interviewing about weight loss will be undertaken to identify participant ambivalence and perceived personal advantages to weight management.

The caloric prescription will be based on $600 \mathrm{kcal}$ below that required for weight maintenance (calculated using the equations for basal metabolic rate according to gender, age and body weight).

Food and drink choices will be based on information obtained from current eating habits obtained through a 24 hour recall questionnaire to guide personalised advice on food frequency, portion sizes and foods to limit. Participant agreed goals (and implementation intentions) will be used for one specific food or drinking habit and this will be self-monitored with personalised feedback provided.

Physical activity dose: all participants will undertake the Scottish Physical Activity Screening Questionnaire ${ }^{37}$ by the coach as an indicator of current activity levels to help guide the communications about walking plans and signpost other activities (including those offered in the local leisure centres). Participant agreed pedometer goals (and implementation intentions) will be used for habitual walking and this will be self-monitored with personalised feedback provided. Participants will be supported to gradually increase physical activity towards accumulating at least $150 \mathrm{~min}$ of moderate intensity physical activity per week and then, where appropriate, towards $300 \mathrm{~min}$ per week (based on Scottish Intercollegiate Guidelines Network $^{38}$ guidance on weight management).

A detailed description of each intervention visit and telephone calls are provided in online supplementary appendix 2.

Intervention delivery: there will be two face-to-face intervention meetings (one of $60 \mathrm{~min}$ and one of $45 \mathrm{~min}$ ). Over a 12-month period there will be up to nine further contacts by telephone. The intervention programme will be delivered by BCN volunteers who have received the training programme to become lifestyle coaches.

Intervention fidelity: we will undertake independent analysis of fidelity to the intervention. Fidelity of programme delivery and content will be assessed by audio-recording and transcription from a purposeful sample of approximately $10 \%$ of ActWELL lifestyle coaches including faceto-face interactions and telephone contacts at each site. A researcher independent from the intervention delivery will analyse the transcripts and evaluate the proportion of points covered in each session relative to those described in the intervention protocol. Time for implementation procedures will be recorded by the lifestyle coaches.

\section{Outcome measures}

A full list of measurable outcomes and sources ${ }^{39-42}$ is presented in table 1 .

Data collection will occur at baseline, 12 weeks and 12 months. Data will be collected by the blinded research nurses from the clinical research centres in each location (trained on the study protocol) at baseline and 12 months, and over the telephone at 3 months, and entered directly onto study case report forms and questionnaires. Detailed Standard Operating Procedures will be provided on each aspect of the protocol to ensure consistent methodology is used by all staff.

At baseline, along with the collection of demographic and background (eg, menopausal and smoking status) data by questionnaire, any contraindications to physical activity will also be assessed using the PAR-Q physical activity readiness questionnaire. ${ }^{43}{ }^{44}$ Where required, and in all women aged 70 years, participants will be asked to seek advice from their general practitioner before becoming more physically active. Height will also be measured to the nearest $1 \mathrm{~mm}$ using a calibrated stadiometer, with shoes removed, in order to determine BMI and confirm eligibility.

\section{Co-primary outcomes}

Co-primary outcomes will be change in body weight and change in physical activity by 12 months. Both will be measured as the mean difference between groups at 12 months adjusted for baseline, site and SIMD. ${ }^{29}$ Weight will be measured to the closest $100 \mathrm{~g}$, in light indoor clothing and without shoes, heavy jewellery or pocket contents, using regularly calibrated electronic scales. Physical activity will be objectively measured using thigh worn activPAL accelerometers. These monitors measure free-living sedentary, upright and ambulatory activity 


\begin{tabular}{|c|c|c|c|c|}
\hline Primary outcomes & & Baseline & 12 weeks & 12 months \\
\hline Body weight & Measured using digital body weight scales $(\mathrm{kg})$ & $\mathrm{x}$ & & $\mathrm{x}$ \\
\hline Physical activity & 7 days accelerometry with activPAL (steps) & $\mathrm{X}$ & & $\mathrm{X}$ \\
\hline \multicolumn{5}{|l|}{ Secondary outcomes } \\
\hline $\begin{array}{l}\text { Modes of physical } \\
\text { activity }\end{array}$ & Scottish Physical Activity Screening Questionnaire ${ }^{34}$ & $x$ & $x$ & $x$ \\
\hline Sedentary behaviour & 7 days accelerometry with activPAL (subsample only) & $\mathrm{x}$ & & $\mathrm{x}$ \\
\hline Anthropometric changes & $\begin{array}{l}\text { Body mass index (height and weight), waist } \\
\text { circumference }(\mathrm{cm})\end{array}$ & $x$ & & $\mathrm{x}$ \\
\hline \multirow[t]{2}{*}{ Eating habits } & Questionnaire based on Scottish Health Survey ${ }^{22}$ & $x$ & & $\mathrm{x}$ \\
\hline & Fruit and vegetable intake ${ }^{39}$ & & $x$ & \\
\hline Alcohol intake & Audit C questionnaire ${ }^{40}$ & $\mathrm{x}$ & & $\mathrm{x}$ \\
\hline \multirow[t]{4}{*}{ Psychosocial variables } & Modified brief illness questionnaire $e^{41}$ & $x$ & & $x$ \\
\hline & $\begin{array}{l}\text { Knowledge and beliefs about lifestyle and breast } \\
\text { cancer risk (developed in-house) }\end{array}$ & $\mathrm{x}$ & & $\mathrm{x}$ \\
\hline & $\begin{array}{l}\text { Psychosocial health measures resources (perceived } \\
\text { motivation, awareness, ability, action, monitoring, and } \\
\text { social support around weight management) }\end{array}$ & & & $\mathrm{x}$ \\
\hline & Perceived body weight (developed in-house) & $\mathrm{x}$ & $\mathrm{X}$ & $x$ \\
\hline \multirow[t]{2}{*}{ Economic outcomes } & EQ-5D-5L questionnaire ${ }^{42}$ & $x$ & $x$ & $x$ \\
\hline & $\begin{array}{l}\text { Economic health resource usage (developed by HERU, } \\
\text { University of Aberdeen) }\end{array}$ & $x$ & & $\mathrm{x}$ \\
\hline \multirow[t]{2}{*}{ Cardiovascular risk } & Blood sampling for lipids & $x$ & & $x$ \\
\hline & Blood pressure & $x$ & & $x$ \\
\hline Diabetes risk & Haemoglobin A1c & $x$ & & $x$ \\
\hline
\end{tabular}

and provide data on step count and time spent sedentary. The devices will be fitted by the research nurses to ensure correct placement, and the participants will be asked to wear them at all times (excluding when swimming or bathing) for eight nights to ensure a full seven days of recording. A demonstration and instructions will be provided in case removal is required.

\section{Secondary outcomes}

Secondary outcomes will be changes in waist circumference, modes (types) of physical activity, sedentary behaviour, eating habits, alcohol intake, psychosocial variables, economic outcomes, haemoglobin A1c (HbAlc), non-fasting lipids and non-fasting insulin, blood pressure and cardiovascular disease risk (incorporating blood pressure and lipid measures).

Waist circumference will be measured midway between the iliac crest and lower costal margin using a Seca 201 measuring tape. Modes of physical activity will be determined from a 7-day recall questionnaire, ${ }^{45}$ and sedentary behaviour from analysis of the activPAL data. Questionnaire-based tools will be used to determine eating habits, alcohol intake, psychosocial variables (eg, illness perception, knowledge and beliefs about lifestyle and breast cancer risk and personal health resources, eg, motivation, awareness and social support around weight management, self-monitoring and perceived ability to manage weight) (table 1). In order to determine the economic outcomes of the trial, data on health-related quality of life was collected using the EQ-5D-5L questionnaire, ${ }^{42}$ along with questions on use of NHS healthcare resources (number of primary care contacts, inpatient, outpatient and day cases).

Blood pressure will be measured using a Microlife 3BTO digital blood pressure monitor, or other approved BP monitor in the local clinical research facility, with the participant seated and relaxed. One non-fasted blood sample will be taken per person at both the baseline and 12-month follow-up visits in order to measure HbAlc, non-fasting lipids and non-fasting insulin. Samples will be processed and stored at local clinical research centres and shipped in batches to Glasgow University laboratories for analysis. All samples will be analysed in a blinded and anonymised fashion at the same time to minimise analytical variation, with no individual results being made available to participants.

\section{Delivery outcomes}

In addition to the intervention delivery time recording and fidelity assessments described earlier, delivery will be also be evaluated in terms of engagement (recruitment and retention), using data collected by coaches, exit 
questionnaires and through interviews with coaches and participants to record their experiences.

\section{Process evaluation}

These measures are aimed at identifying feasibility and acceptability issues pertinent to decisions about roll-out:

\section{Breast screening centres}

- Observations from the waiting room by the research team will be conducted on two occasions per site before and after recruitment commences. Data on clinic start and end times will be noted and mammography staff asked to provide comments relating to clinic flow.

- In order to estimate the reach of endorsements by mammographers, we will request that clinic numbers are obtained (preferably as appointments attended), and track total ActWELL cards provided to clinics and total cards returned.

- A sample of mammographers will be invited to participate in individual semi-structured qualitative interviews (one interview per site, total $n=4$ ) to explore perceptions and experiences of recruitment, including perceived ease of study introduction, time burden, positive and negative experiences that have arisen, handling of questions, support from clinic staff and training from research team. Interviews will be conducted shortly after the end of the study recruitment period (eg, September 2018) to enable mammographers to reflect on experiences over the whole period while recall is still relatively fresh.

\section{$\mathrm{BCN}$ are collecting information on}

- advertising for coaches (where, when and costs);

- responses to advertising (applications and telephone/ email queries);

- time and volunteer/coach experience of joint training programme with ActWELL team;

- volunteer coach reported problems, queries and reasons for drop out;

- coordinators' experience of managing coaches.

These data will be used to inform the economic analysis.

BCN lifestyle coaches will be asked to collect data on participant contacts

- Number of face-to-face visits attended.

- Number of phone calls achieved.

- Time taken for each contact.

- Perceived engagement at each contact.

- A sample of coaches from each site (two per site, total $n=8$ ) will be invited to participate in individual semi-structured qualitative interviews to explore perceptions and experiences of delivery, including recruitment, training, implementation, participant contact procedures, perception of intervention acceptability, time commitments and exit strategies. The interviews will also explore coaches' perceptions of facilitators and barriers to participant engagement in the programmes. Interviews will be conducted shortly after the end of the delivery period.

\section{Leisure centres}

- A sample of four leisure centre coordinators will be invited to participate in individual semi-structured qualitative interviews to explore their perceptions and experiences of hosting the coach sessions, including burden, space, time, challenges and any potential benefits or negative consequences for the leisure centre. Interviews will be conducted shortly after the end of the delivery period (eg, May 2019) to enable coordinators to reflect on experiences over the whole period while recall is still relatively fresh.

\section{Participants}

Semi-structured qualitative interviews will be conducted with 20 intervention participants (5 per study site) after expression of an interest to participate during the final 12 -month visit. Interviews will be conducted via telephone. Interviews will be conducted at the end of each participant's period of engagement in the study to allow participants to reflect over the entire period of their engagement while recall is still relatively fresh. We decided not to interview participants during their engagement in the study so as to avoid introducing any intervention effects which might have arisen from participants being sensitised to the questions and issues under discussion.

A semi-structured interview schedule designed to cover key topics while also eliciting additional participant perspectives will be used. Interviews will cover participants' views and experiences of engaging with the study, including motives for participation and understanding of the study purpose; possible impact of the study on attendance for screening; perceptions and opinions of the programme content, duration, accessibility intensity, recruitment and exit strategy; views of the coaching process and experience of setting goals; experiences of attempting to make changes over the 12-month intervention period, and the facilitators and barriers to making changes. Possible facilitators and barriers to be explored include personal beliefs, motivation, family members, social and coach support. With the consent of participants, all interviews will be audio-recorded.

\section{STATISTICAL CONSIDERATIONS Sample size}

Body weight: using the data from the overweight women $\left(\mathrm{BMI}>25 \mathrm{~kg} / \mathrm{m}^{2}\right.$ ) in the ActWELL feasibility study (mean body weight $80.9 \pm 17.9 \mathrm{~kg}$ ), a total of 414 women (207 per group) would be needed to detect a $7 \%$ wt change at $90 \%$ power. Allowing for $25 \%$ drop out (based on our findings from the feasibility study), this would mean randomising 552 women. Based on feasibility data, we estimate that we would need 849 women to express an interest in the study which allows for $25 \%$ who would be ineligible on grounds of $\mathrm{BMI}<25 \mathrm{~kg} / \mathrm{m}^{2}$ and $10 \%$ who initially express 
interest then change their minds. The NHSSBSP screens thousands of women each year so we do not anticipate any problems with 849 women expressing an interest in the study.

Physical activity: given that the activity data from the ActWELL feasibility study was based on self-report, an objective measurement of steps using pedometers in the predominantly $(80 \%)$ female participants of the 'Walking for Well-being in the West' study ${ }^{45}$ was used to inform the sample size calculation. At baseline, a mean of $6802 \pm 3212$ steps were recorded in the intervention group. In order to detect a difference of 2000 steps between groups at follow-up, at $90 \%$ power, 102 women (51 per group) would be required to complete this aspect of the study using activPAL accelerometers (shown to be feasible in studies of older adults and enabling accurate estimates of activity and sedentary behaviour ${ }^{46}{ }^{47}$ ). Allowing for $20 \%$ drop out plus any equipment malfunction/postal losses, we would recruit a further $30 \%$ bringing the total enrolment to 146 of the 552 participants for this aspect of the study. In summary, a subsample of 146 of the 552 women recruited above will be asked to wear the accelerometers.

\section{Quantitative analysis}

Statistical analysis of outcomes will be undertaken by statisticians at TCTU. In the main analysis, we will use an intention-to-treat analysis with all available data. We will undertake multiple linear regression analyses with mixed effects models adjusted for the corresponding baseline values with group allocation and site as fixed effects. For the primary outcomes, we will also undertake a sensitivity analysis using both repeated measures and multiple imputations for missing values.

We do not anticipate that clustering effects will be a substantial issue in ActWELL, which is why we have designed the trial as individually randomised. We anticipate 24 or more BCN lifestyle coaches delivering the intervention, making each cluster small at around 10 participants. It is possible that even more lifestyle coaches will be involved depending on the capacity of BCN. We also anticipate that participants will be evenly distributed across the four breast screening centres rather than having a dominant centre. We will, however, look for evidence of clustering effects as a secondary analysis and present and interpret our results in light of this analysis.

\section{Missing data}

The extent of missing data will be examined and, if necessary, methods such as multiple imputation will be implemented to provide robust results, assuming data are missing at random (MAR). We will examine the extent of missing data by considering the differences between those with complete data and those with missing data, and if they are similar we will assume data are missing completely at random. If they do differ we will assume MAR and use multiple imputations and compare with the primary analysis. We will try to obtain reasons for missing data during the trial and if the probability of missing data are related to the outcome then data may not be missing at random. If this looks likely, then mechanisms of missingness and outcomes will be modelled together.

\section{Qualitative analysis}

All interview recordings will be transcribed in full for analysis. Transcripts will be coded for thematic analysis, with the coding themes to be agreed among the researchers based on the core questions and topic areas, including any new and emerging themes. The reliability of the themes will then be reassessed by a process of familiarisation with the transcript texts. Discussions between researchers will enable identification of emerging themes and resolution of interpretive difference.

\section{Cost-effectiveness analysis}

Intervention costs data will be collected by health economics team including:

- BCN costs (coordinator salary, training, transport);

- Intervention consumable costs (training packs and participant materials);

- Intervention delivery staff costs (coach training, mentoring and overseeing costs).

The cost-effectiveness analysis of the ActWELL intervention compared with usual care will be based on resource use and outcomes (EQ-5D-5L) data ${ }^{42}$ (collected from participants' questionnaires and telephone interviews). This will take the format of a within-trial cost-effectiveness analysis and use a cost-utility analysis framework. The effects of the ActWELL intervention will be estimated as gain in quality-adjusted life years (QALYs) at 12 months using EQ-5D-5L data collected at baseline, 3 and 12 months. Estimates of cost-effectiveness will be expressed as the incremental cost per QALY gained (over 12 months).

\section{Patient and public involvement}

The development of research questions and outcome measures arose from pilot work, ${ }^{27}$ which included feedback from participants. A public advisory group was established comprising three patient representatives from breast screening attendees recruited by BCN, plus a patient advisor on the investigation team (who will chair the public advisory group). The public advisory group will be involved in every aspect of the trial. This is particularly important with respect to trial design and feedback, issues including inequalities, access to intervention, recruitment, perspectives on written, verbal and e-communications, burden of study procedures (including questionnaires), interpretation of qualitative data, conduct of the study and dissemination events. In addition, information attained from formative research with screening participants will be used to assist in the design of the intervention content and delivery. After the study is complete, participants will be interviewed to gain insight into the study burden and procedures. A summary of results will be available for all participants. 


\section{Ethics and dissemination}

The ActWELL trial follows all procedures set out by the Tayside clinical trials unit including reporting adverse and serious adverse events. All reports will be reviewed by the senior clinician and appropriate actions taken. The intervention is considered low risk and all coaches are trained on supporting modest changes in physical activity to decrease risks associated with vigorous or intense activity.

The study dissemination plan includes reports to the funder to assist in decision making about potential roll out. Reports of the findings will also be shared with BCN, Scottish Screening Committee, Scottish Cancer Task Force, participating screening centres, participating leisure centres and our public advisory group. A summary of findings will be available for all participants.

Academic dissemination through papers in peer-reviewed journals and conference presentations will be focused on reporting the main impact of the intervention on primary and secondary outcomes, health economic evaluation and qualitative findings that will assist decisions about roll out. Findings related to recruitment challenges, the experience of volunteer coaches and psychosocial findings which may have impacted on process and outcomes will also be reported.

Public dissemination will use social media channels including those of the Scottish Cancer Prevention Network, academic institutions involved and public events such as 'café science'.

\section{DISCUSSION}

This paper describes the protocol for a randomised controlled trial to determine the effectiveness and cost-effectiveness of a weight management intervention targeted at women aged between 50 and 70 years of age who are invited to attend routine breast screening clinics. This is consistent with the concept that 'every healthcare contact is a health improvement opportunity' as embodied within NHS Scotland's health promoting health service. ${ }^{48}$

Current epidemiological evidence highlights adult weight gain, excess body fat, low levels of physical activity and alcohol intake as modifiable factors associated with an increased risk of developing postmenopausal breast cancer. ${ }^{3}$ The NHS breast screening setting is one of only a few nationwide opportunities that offers face-to-face contact by a health professional to over $70 \%$ of (healthy) women aged 50-70 years including significant numbers of women from deprived areas $(>60 \%$ of women from SIMD quintile 1 and $>70 \%$ from quintile 2$)^{26}$ who may have no other opportunity to access free, personalised lifestyle change support. Few other free, national NHS clinical services provide a routine invitation to reach this number of women in this age group. At present, no support for lifestyle change is provided in this setting, although survey and intervention work highlights the potential for engagement and behaviour change. ${ }^{19} 28$ Recent work undertaken in Scotland in routine breast screening clinics has reported that a simple women's magazine intervention about breast cancer risk produced by The Scottish Cancer Prevention Network resulted in $60 \%$ of respondents claiming an increased knowledge about breast cancer, lifestyle and motivation to find out more about cancer prevention with $40 \%$ of respondents expressing intentions to make lifestyle changes. ${ }^{49}$ It was notable that there was no difference in results by social position. While concerns have been raised about the potential for increasing health inequalities through individual level interventions, a recent Cochrane review has reported that individual obesity management interventions (in both children and adults) do not increase health inequalities. $^{50}$

While the current trial has primary outcomes of increased physical activity and weight loss, the programme will target energy expenditure (caloric usage in physical activity) and dietary intake (caloric intake from foods, soft and alcoholic drinks). The length of the study is designed to embrace the principles of weight loss and weight loss maintenance and therefore has the potential to achieve a long-term change in lifestyle habits. The study has been developed from a well-conducted feasibility trial with positive indicative outcomes and uses a gold standard multicentre, randomised controlled design methodology. The development of the intervention used a unique combination of staff from academia, NHS, breast cancer charity and healthy screening attendees and employs a novel approach to deliver weight management by volunteers in community locations. The study is not powered to assess long-term risk of breast cancer and indeed the intervention would need to demonstrate effective weight loss prior to investment in a cancer outcomes trial. There have been no trials of primary prevention of breast cancer using a weight management intervention (largely due to length of follow-up and study size). However, there are a number of ongoing trials of weight management interventions in women with a breast cancer diagnosis which will report on cancer and all-cause mortality end points (as described by Demark-Wahnefried $e t a l^{\tilde{p}^{1}}$ ).

It should be noted that the trial is also designed to collect information relevant for clinicians and policymakers responsible for considerations about the potential roll out of the programme throughout the national NHS breast screening programme drawing on feedback from screening staff and participants. This trial is highlighted within the National Cancer Strategy as an investment from Scottish government in cancer prevention. ${ }^{52}$ The programme may also have salience internationally where population-based breast cancer screening programmes are offered.

\section{Author affiliations}

${ }^{1}$ Division of Cancer Research, Centre for Research into Cancer Prevention and Screening, Division of Population Health and Genomics, University of Dundee, Ninewells Hospital \& Medical School, Dundee, UK

${ }^{2}$ Physical Activity for Health Research Centre, Institute for Sport, Physical Education and Health Sciences, University of Edinburgh, Edinburgh, UK

${ }^{3}$ Department of Breast Surgery, NHS Tayside, Ninewells Hospital \& Medical School, Dundee, UK 
${ }^{4}$ Health Economics Research Unit, University of Aberdeen, Aberdeen, UK ${ }^{5}$ Division of Psychology, School of Natural Sciences, University of Stirling, Stirling, UK

${ }^{6}$ Tayside Clinical Trials Unit, Tayside Medical Sciences Centre, Ninewells Hospital and Medical School, Dundee, UK

${ }^{7}$ Institute of Cardiovascular and Medical Sciences, University of Glasgow, Glasgow, UK

${ }^{8}$ Institute for Social Marketing, University of Stirling, Stirling, UK

${ }^{9}$ Health Services Research Unit, University of Aberdeen, Aberdeen, UK

Acknowledgements The authors would like to acknowledge the assistance of Mary Allison, Amy Hickman and Eluned Hughes from Breast Cancer Now and Dr Stephen Caswell in manuscript preparation and coordination of volunteer training. Staff at the NHS Scottish Breast Screening Service who have assisted in programme design. The authors would also like to acknowledge our patient advisor (Elspeth Banks) and Public Advisory Group members (Pamela Deponio, Maggie Taylor and Mary Wotherspoon) for their regular communications and valuable time. The Health Services Research Unit and The Health Economics Research Unit, University of Aberdeen receives core funding from the Chief Scientist Office of the Scottish Government Health Directorates.

Contributors ASA is the principal investigator and has overall responsibility for all aspects of the ActWELL study. She initiated the study, designed the intervention, led on development of the protocol and drafting the manuscript. AMC, CM and NM were involved in intervention design and training, assessment methodology, finalising the protocol, reading, editing and approving the manuscript. SG is the trial manager and responsible for coordinating all aspects of the ActWELL study, drafting the protocol and reading, editing and approving the manuscript. EJM has responsibility for clinical issues, inclusion and exclusion criteria, trial design, finalising the protocol and reading, editing and approving the manuscript. ARN is responsible for health economics analysis, assessment methodology, finalising protocol and reading, editing and approving the manuscript. REO'C has responsibility for all psychological aspects of intervention design, assessment methodology, fidelity measures, finalising the protocol and reading, editing and approving the manuscript. PR is responsible for statistical design, analysis plan drafting protocol and reading, editing and approving the manuscript. NS is responsible for design of blood collection procedures, overseeing analysis of bloods, finalising protocol design and reading, editing and approving the manuscript. MS is responsible for formative qualitative analysis, intervention design, acceptability measures, finalising protocol and reading, editing and approving the manuscript. ST is responsible for trial design, recruitment strategies, day-to-day management decisions (with ASA and SG), finalising protocol reading, editing and approving the manuscript.

Funding This work was supported by The Scottish Government, grant number BC/ Screening/17/01.

\section{Competing interests None declared.}

Patient consent Not required.

Ethics approval Ethical approval for this study was provided by East of Scotland Research Ethics Service (REC reference: 17/ES/0073). Our initial submission to ethics in January 2017 was withdrawn as we were advised to submit the formative work to the University of Dundee ethics committee (approved 5 April 2017, Ref 020/17) and the remainder to NHS ethics once the formative work was completed. It took several months to complete the formative work which delayed both the NHS ethics submission (approval provided on 22 June 2017) and the recruitment start date. This resulted in a delay in registering the study with ISCRTN until the final protocol was completed and the submission details updated. ISCRTN registration was assigned prospectively on 21 July 2017. Recruitment for the study commenced on 1 August 2017 with first participant randomised on 5 September 2017 due to the aforementioned project delay.

Provenance and peer review Not commissioned; externally peer reviewed.

Data sharing statement The authors are happy to provide data upon request to the corresponding author.

Open access This is an open access article distributed in accordance with the Creative Commons Attribution Non Commercial (CC BY-NC 4.0) license, which permits others to distribute, remix, adapt, build upon this work non-commercially, and license their derivative works on different terms, provided the original work is properly cited, appropriate credit is given, any changes made indicated, and the use is non-commercial. See: http:// creativecommons.org/licenses/by-nc/4.0/.

\section{REFERENCES}

1. National Services Scotland. Cancer Incidence in Scotland (2014). https://www.isdscotland.org/Health-Topics/Cancer/Publications/ 2016-05-17/2016-05-17-Cancer-Incidence-Summary.pdf (Accessed 15 Jan 2018).

2. National Services Scotland. Cancer incidence projections for Scotland. http://www.isdscotland.org/Health-Topics/Cancer/ Publications/2015-08-18/2015-08-18-Cancer-Incidence-ProjectionsReport.pdf (Accessed 15 Jan 2018)

3. WCRF. Cancer statistics. https://www.wcrf-uk.org/uk/preventingcancer/cancer-preventability-statistics/breast-cancer (Accessed 15 Jan 2018).

4. World Cancer Research Fund International / American Institute for Cancer Research, 2017. Continuous update project report: diet, nutrition, physical activity and breast cancer wcrf.org/breast-cancer2017 (Accessed 15 Jan 2018).

5. Eliassen AH, Colditz GA, Rosner B, et al. Adult weight change and risk of postmenopausal breast cancer. JAMA 2006;296:193-201.

6. Ahn J, Schatzkin A, Lacey JV, et al. Adiposity, adult weight change, and postmenopausal breast cancer risk. Arch Intern Med 2007;167:2091-167.

7. Emaus $\mathrm{MJ}$, van Gils $\mathrm{CH}$, Bakker MF, et al. Weight change in middle adulthood and breast cancer risk in the EPIC-PANACEA study. Int $J$ Cancer 2014;135:2887-99.

8. Neuhouser ML, Aragaki AK, Prentice RL, et al. Overweight, Obesity, and Postmenopausal Invasive Breast Cancer Risk: A Secondary Analysis of the Women's Health Initiative Randomized Clinical Trials. JAMA Oncol 2015;1:611-21.

9. Hastert TA, Beresford SA, Patterson RE, et al. Adherence to WCRF/AICR cancer prevention recommendations and risk of postmenopausal breast cancer. Cancer Epidemiol Biomarkers Prev 2013;22:1498-508.

10. Kohler LN, Garcia DO, Harris RB, et al. Adherence to diet and physical activity cancer prevention guidelines and cancer outcomes: a systematic review. Cancer Epidemiol Biomarkers Prev 2016;25:1018-28.

11. Byers T, Sedjo RL. Does intentional weight loss reduce cancer risk? Diabetes Obes Metab 2011;13:1063-72.

12. Schauer DP, Feigelson HS, Koebnick C, et al. Bariatric Surgery and the Risk of Cancer in a Large Multisite Cohort. Ann Surg 2017:1.

13. Lauby-Secretan $B$, Scoccianti $C$, Loomis $D$, et al. Body fatness and cancer-viewpoint of the IARC Working Group. N Engl J Med 2016;375:794-8.

14. Lean ME, Leslie WS, Barnes AC, et al. Primary care-led weight management for remission of type 2 diabetes (DiRECT): an openlabel, cluster-randomised trial. Lancet 2018;391:541-51.

15. Owens $\mathrm{C}$, Conaghan $\mathrm{PG}$. Improving joint pain and function in osteoarthritis. Practitioner 2016;260:17-20.

16. National Services Scotland. Scottish breast screening programme. http://www.isdscotland.org/Health-Topics/Cancer/Publications/201704-25/2017-04-25-SBSP-Cancer-Report.pdf

17. Fisher B, Dowding D, Pickett KE, et al. Health promotion at NHS breast cancer screening clinics in the UK. Health Promot Int 2007;22:137-45.

18. Fisher BA, Wilkinson L, Valencia A. Women's interest in a personal breast cancer risk assessment and lifestyle advice at NHS mammography screening. J Public Health 2017;39:113-21.

19. Anderson AS, Mackison D, Boath C, et al. Promoting changes in diet and physical activity in breast and colorectal cancer screening settings: an unexplored opportunity for endorsing healthy behaviors. Cancer Prev Res 2013;6:165-72.

20. Eccles SA, Aboagye EO, Ali S, et al. Critical research gaps and translational priorities for the successful prevention and treatment of breast cancer. Breast Cancer Res 2013;15:R92.

21. Howell A, Anderson AS, Clarke RB, et al. Risk determination and prevention of breast cancer. Breast Cancer Res 2014;16:446.

22. Scottish Government, 2017. Scottish health survey: main report [online] http://www.gov.scot/Publications/2017/10/2970/downloads (Accessed 15 Jan 2018)

23. NICE. Weight management: lifestyle services for overweight or obese adults. Public health guideline [PH53]. https://www.nice.org.uk/ guidance/ph53

24. Dietz WH, Baur LA, Hall K, et al. Management of obesity: improvement of health-care training and systems for prevention and care. Lancet 2015;385:2521-33.

25. NHS. Breast screening. http://www.nsd.scot.nhs.uk/services/ screening/breastscreening/index.htm

26. NHS. Scottish breast screening programme. http://www.isdscotland. org/Health-Topics/Cancer/Breast-Screening/

27. Anderson AS, Macleod M, Mutrie N, et al. Breast cancer risk reduction-is it feasible to initiate a randomised controlled trial of a 
lifestyle intervention programme (ActWell) within a national breast screening programme? Int J Behav Nutr Phys Act 2014;11:156.

28. Conway $\mathrm{E}$, Wyke S, Sugden J, et al. Can a lifestyle intervention be offered through NHS breast cancer screening? Challenges and opportunities identified in a qualitative study of women attending screening. BMC Public Health 2016;16:758.

29. Scottish Government. Scottish index of multiple deprivation. http:// www.scotland.gov.uk/Topics/Statistics/SIMD/ (accessed 19 Jan 2018).

30. Breast Cancer Now, 2017. Breast cancer risk the Facts http:// breastcancernow.org/sites/default/files/public/risk_booklet_pdf_final_ sept 2015 1.pdf (accessed 30 Mar 2017).

31. Anderson AS, Dunlop J, Gallant S, et al. Feasibility study to assess the impact of a lifestyle intervention ('LivingWELL') in people having an assessment of their family history of colorectal or breast cancer. BMJ Open 2018;8:e019410.

32. Michie S, Abraham C, Whittington C, et al. Effective techniques in healthy eating and physical activity interventions: a meta-regression. Health Psychol 2009;28:690-701.

33. Michie S, van Stralen MM, West R. The behaviour change wheel: a new method for characterising and designing behaviour change interventions. Implement Sci 2011;6:42.

34. Michie S, Ashford S, Sniehotta FF, et al. A refined taxonomy of behaviour change techniques to help people change their physical activity and healthy eating behaviours: the CALO-RE taxonomy. Psychol Health 2011;26:1479-98.

35. Knowler WC, Barrett-Connor E, Fowler SE, et al. Reduction in the incidence of type 2 diabetes with lifestyle intervention or metformin. N Engl J Med 2002;346:393-403.

36. Beeken RJ, Leurent $B$, Vickerstaff $V$, et al. A brief intervention for weight control based on habit-formation theory delivered through primary care: results from a randomised controlled trial. Int J Obes 2017;41:246-54.

37. Physical Activity and Health Alliance. Scottish physical activity screening question (Scot-PASQ). 2013. Available at http://www.paha. org.uk/Resource/scottish-physical-activity-screening-question-scotpasq (Accessed 30 Mar 2018).

38. Scottish Intercollegiate Guidelines Network (SIGN). Management of obesity - a national clinical guidelines 115. Edinburgh: Royal College of Physicians Edinburgh, 2010.

39. Cappuccio FP, Rink E, Perkins-Porras L, et al. Estimation of fruit and vegetable intake using a two-item dietary questionnaire: a potential tool for primary health care workers. Nutr Metab Cardiovasc Dis 2003;13:12-19.
40. Bush K, Kivlahan DR, McDonell MB, et al. The AUDIT alcohol consumption questions (AUDIT-C): an effective brief screening test for problem drinking. Ambulatory Care Quality Improvement Project (ACQUIP). Alcohol Use Disorders Identification Test. Arch Intern Med 1998;158:1789-95.

41. Broadbent E, Petrie KJ, Main J, et al. The brief illness perception questionnaire. J Psychosom Res 2006;60:631-7.

42. EuroQol Group. EuroQol - a new facility for the measurement of health-related quality of life. Health Policy 1990;16:199-208.

43. Thomas S, Reading J, Shephard RJ. Revision of the Physical Activity Readiness Questionnaire (PAR-Q). Can J Sport Sci 1992:17:338-45.

44. Goodman JM, Thomas SG, Burr J. Evidence-based risk assessment and recommendations for exercise testing and physical activity clearance in apparently healthy individuals. Appl Physiol Nutr Metab 2011;36(Suppl 1):S14-32.

45. Lowther M, Mutrie N, Loughlan C, et al. Development of a Scottish physical activity questionnaire: a tool for use in physical activity interventions. Br J Sports Med 1999;33:244-9.

46. Baker G, Gray SR, Wright A, et al. The effect of a pedometer-based community walking intervention "Walking for Wellbeing in the West" on physical activity levels and health outcomes: a 12-week randomized controlled trial. Int J Behav Nutr Phys Act 2010;7:51.

47. Mutrie N, Doolin O, Fitzsimons CF, et al. Increasing older adults walking through primary care: results of a pilot randomized controlled trial. Fam Pract 2012;29:633-42.

48. Health Promoting Health Service Service- NHS Health Scotland, 2009. Overview www.healthscotland.com/documents/4128.aspx

49. Macleod M, Anderson AS. Cancer prevention-the feasibility and acceptability of promoting breast cancer risk reduction in the screening setting through a lifestyle magazine. Eur J Cancer Care 2018;27:e12823.

50. Bambra CL, Hillier FC, Cairns JM, et al. Summerbell CD How effective are interventions at reducing socioeconomic inequalities in obesity among children and adults? Two systematic reviews. Southampton (UK): NIHR Journal Library, 2015.

51. Demark-Wahnefried W, Schmitz KH, Alfano CM, et al. Weight management and physical activity throughout the cancer care continuum. CA Cancer J Clin 2018;68:64-89.

52. Scottish Government, 2016. Beating cancer: ambition and action Scottish Government http://www.gov.scot/Publications/2016/03/ 9784 